\title{
Non-axisymmetric accretion on the classical TTS RW Aur A*
} \author{
M. T. V. T. Lago ${ }^{3,4}$, and I. Tuominen ${ }^{1}$ \\ 1 Astronomy Division, PO Box 3000, 90014 University of Oulu, Finland \\ 2 Stockholm Observatory, 13336 Saltsjöbaden, Sweden \\ 3 CAUP, Universidade do Porto, Rua das Estrelas, 4150 Porto, Portugal \\ ${ }^{4}$ Departamento de Matemática Aplicada, Rua das Taipas, 135, 4150 Porto, Portugal
}

P. P. Petrov ${ }^{1, \star \star}$, G. F. Gahm ${ }^{2}$, J. F. Gameiro ${ }^{3,4}$, R. Duemmler ${ }^{1}$, I. V. Ilyin ${ }^{1}$, T. Laakkonen ${ }^{1}$,

Received 7 September 2000 / Accepted 1 February 2001

\begin{abstract}
High-resolution spectroscopic monitoring of the exceptionally active classical T Tauri star (CTTS) RW Aur A was carried out in three seasons of 1996, 1998 and 1999 with simultaneous $B, V$ photometry. The high quality spectra revealed a multicomponent structure of the spectrum, which includes: 1) a veiled photospheric spectrum of a K1-K4 star, 2) broad emission lines of neutrals and ions, 3) narrow emission lines of He I and He II, 4) red-shifted accretion features of many lines, 5) shell lines at about the stellar velocity, 6) blue-shifted wind features and 7) forbidden lines.

Periodic modulations in many spectral features were found. The photospheric absorption lines show sinusoidal variations in radial velocity with an amplitude of $\pm 6 \mathrm{~km} \mathrm{~s}^{-1}$ and a period of about 2 d. 77 . The radial velocities of the narrow emission lines of He vary with the same period but in anti-phase to the photospheric lines. The equivalent widths of the narrow emissions vary with a phase-shift with respect to the velocity curve. The strength of the red-shifted accretion components of $\mathrm{Na} \mathrm{D}$ and other lines is also modulated with the same period. The broad emission lines of metals vary mostly with the double period of about 5.5 .

One unexpected result is that no correlation was found between the veiling and the brightness, although both parameters varied in wide ranges. This is partly due to a contribution of the shell absorption to the photospheric line profiles, which make them vary in width and depth thus simulating lower veiling.

The spectral lines of the accreting gas show two distinct components: one is formed at low velocity at the beginning of the accretion column, and the other at high velocity near the stellar surface. The low velocity components are strong in low excitation lines of neutrals, while the high velocity components are strong in high excitation lines of ions, thus showing the gradients of temperature and density along the accretion column.

Most of the observed features can be interpreted in the framework of non-axisymmetric magnetospheric accretion, but the origin of this asymmetry can be explained in different ways. We consider two possible models. The first model suggests that RW Aur A is a binary with a brown dwarf secondary in a nearly circular orbit with a period of 2 . 77 . The orbiting secondary generates a moving stream of enhanced accretion from one side of the disk towards the primary. The other model assumes that RW Aur A is a single star with a rotational period of 5.5 and with two footpoints of channeled accretion streams within a global magnetosphere which is tilted relative to the rotational axis or otherwise non-axisymmetric. Both models can explain qualitatively and quantitatively most of the observed variations, but there are some details which are less well accounted for.
\end{abstract}

Key words. stars: individual: RW Aur A - stars: pre-main sequence - stars: circumstellar matter - accretion stars: variables

Send offprint requests to: P. P. Petrov, e-mail: Peter.Petrov@Oulu.Fi

* Based on observations made with the Nordic Optical Telescope, operated on the island of La Palma jointly by Denmark, Finland, Iceland, Norway, and Sweden, in the Spanish Observatorio del Roque de los Muchachos of the Instituto de Astrofisica de Canarias.

${ }^{\star \star}$ on leave from the Crimean Astrophysical Observatory.

\section{Introduction}

RW Aurigae is one of the brightest T Tauri stars (TTSs). It is also a most unusual and extreme object of its kind, and has always played a joker in the exploration of the nature of young low mass pre-main sequence objects.

RW Aur is a resolved triple star system (Ghez et al. 1993), and has a bipolar jet rooted at the brightest component, RW Aur A (e.g. Mundt \& Eislöffel 1998; Dougados et al. 2000). Strong and rapid, but irregular brightness 
variations are common - usually measured for component A, which dominates the flux (see e.g. Gahm et al. 1993; Herbst et al. 1994), and the star is a bright infrared source, with a rather flat spectrum longward of $1 \mu \mathrm{m}$ (Cohen \& Schwartz 1976; Ghez et al. 1997).

Already Joy (1945) commented on the magnificent spectrum with strong and broad emission lines. Weak, presumably photospheric, absorption lines indicate a star of spectral type K (e.g. Mundt \& Giampapa 1982; Valenti et al. 1993), and Hartmann et al. (1986) found that these lines vary in radial velocity. The weakness of the lines indicates the presence of continuous and/or line excess emission - the spectrum is veiled.

The emission line spectrum is very complex, and many lines are blended with each other. Some of the broad emission lines also have narrow emission components, and some have absorption components: broad red-shifted, central and/or slightly blue-shifted in velocity. All these components have been found to undergo strong and rapid (hours/days) changes in fluxes, profiles, and radial velocities (Gahm 1970; Appenzeller \& Wolf 1982; Hartmann 1982; Mundt \& Giampapa 1982; Appenzeller et al. 1983; Grinin et al. 1983; Stout-Batalha \& Batalha 2000).

Many ideas on the cause of the photometric and spectral variability observed on classical TTSs, like RW Aur A, have seen light over the years. Presently, the concept of magnetospheric accretion has been successful in explaining many observed phenomena (see Hartmann 1998, and references therein). Irregular accretion to heated regions at the stellar poles has been recognized as a process for producing irregular light variability (variable veiling hot spots), while gas blobs flowing through the magnetosphere could cause variability of the emission line spectrum (see e.g. Gullbring et al. 1996 and references therein).

The magnetospheric accretion model predicts a correlation between veiling and brightness. Such correlations have been reported (e.g. Gahm et al. 1995; Hessman \& Guenther 1997; Chelli et al. 1999), but rather little systematic, long-term spectroscopic and photometric monitoring has been made in order to explore this effect.

The original aim of the present program was to check this particular prediction. One of the selected objects was RW Aur A, which shows clear evidence of variable accretion in several spectral lines along with the chaotic photometric variability. Surprisingly, we found practically no correlation between the degree of veiling and the brightness, as will be discussed below. Instead, we discovered periodic radial velocity changes in the photospheric lines, and also periodic phenomena in practically all other spectral components (Gahm et al. 1999, hereafter called Paper I).

In Paper I, we focussed on the possibility that RW Aur A is a close binary with a secondary of very low mass, possibly a brown dwarf, moving in a nearly circular orbit at less than 10 solar radii from the primary. We reserved a full discussion of all spectral features, and their complex and interwoven variabilities, for a more extensive presentation. In addition, we have also collected a

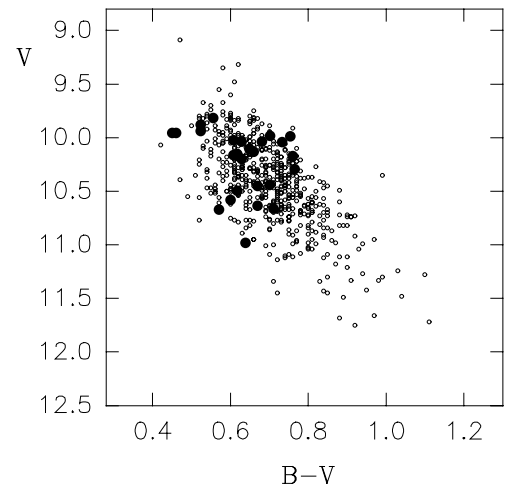

Fig. 1. Colour-magnitude diagram. Open circles: data from the Herbst catalogue; filled circles: our observations

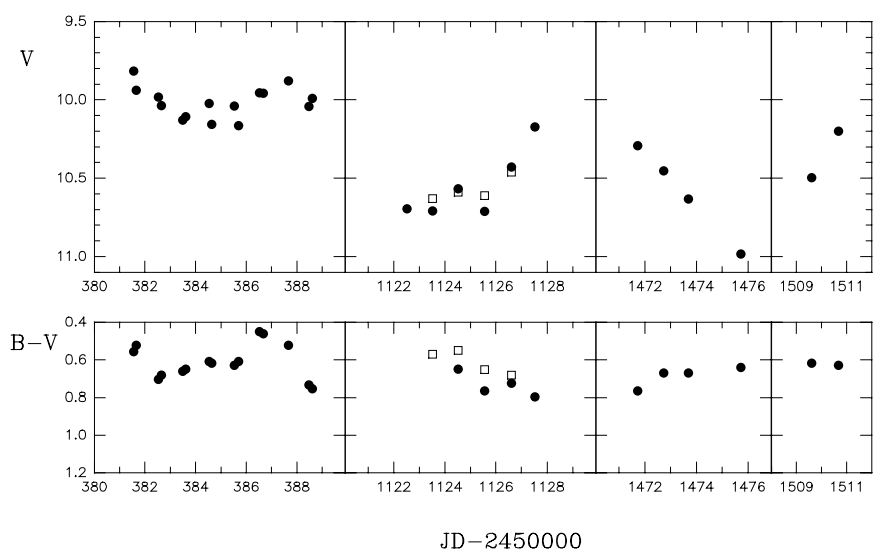

Fig. 2. Light and colour curves for the seasons 1996, 1998, and 1999. Filled circles: CCD observations at NOT; open squares: photoelectric observations at the Swedish telescope

new series of observations of the star at the Nordic Optical Telescope in 1999. The present paper summarizes our findings.

\section{Observations}

High-resolution spectra were collected with the SOFIN échelle spectrograph (Tuominen et al. 1999) at the $2.56 \mathrm{~m}$ Nordic Optical Telescope (NOT) during several observing periods in 1995, 1996, 1997, 1998 and 1999 (Table 1). We used the 3rd camera, which provides a spectral resolution of about $12 \mathrm{~km} \mathrm{~s}^{-1}$ with the entrance slit of $1^{\prime \prime}$.7 ( $R=26000$ ). The secondary components (RW Aur B and $\mathrm{C}$ ) were mostly outside of the entrance slit, but when the seeing was not good enough, the secondary could contribute to the observed spectrum. The flux ratio of the components A/BC is 18 at $5550 \AA$ and 12 at $6750 \AA$ (Ghez et al. 1997). From this, we can estimate the contribution from the secondary stars as a few percent (in the red continuum) in the worst case. In the night of best seeing we took one spectrum of RW Aur BC separately. The only strong emission line is $\mathrm{H} \alpha$, and the lines of $\mathrm{He}$ I and Ca II are weak and narrow. All these lines are very much stronger in the spectrum of RW Aur A. We conclude that 
Table 1. The first 3 columns constitute the observing log. SNR is the signal-to-noise ratio in the continuum near H $\alpha$. The next 4 columns present the radial velocities for the weak absorption lines (WAL) and the He I narrow emission line together with their standard deviations $(\sigma)$; all velocities are in the stellar restframe, i.e. the mean stellar radial velocity of $+16 \mathrm{~km} \mathrm{~s}^{-1}$ has been subtracted. Columns 9 and 10 give the veiling factor and its standard deviation. The last 3 columns give the simultaneous $U B V$ photometry

\begin{tabular}{|c|c|c|c|c|c|c|c|c|c|c|c|c|}
\hline Date & HJD $245 \ldots$ & file & SNR & WAL & $\sigma_{\mathrm{WAL}}$ & He I & $\sigma_{\mathrm{HeI}}$ & veil & $\sigma_{\text {veil }}$ & $\bar{V}$ & $B-V$ & $U-B$ \\
\hline 03/04 Dec 95 & 0055.521 & 06249 & 120 & +4.8 & 1.6 & +3 & 1.0 & 3.9 & 0.7 & - & - & - \\
\hline 05/06 Dec 95 & 0057.565 & 06424 & 120 & +8.8 & 2.8 & +7 & 2.0 & 3.1 & 0.5 & - & - & - \\
\hline $24 / 25$ Oct 96 & 0381.586 & 07032 & 140 & +3.5 & 1.4 & +11 & 3.0 & 4.6 & 0.7 & 9.82 & 0.55 & - \\
\hline $24 / 25$ Oct 96 & 0381.631 & 07036 & 130 & +0.8 & 1.4 & +15 & 2.0 & 3.4 & 0.4 & 9.94 & 0.52 & — \\
\hline $25 / 26$ Oct 96 & 0382.570 & 07097 & 200 & -1.0 & 2.0 & 0 & 2.0 & 7.0 & 2.0 & 9.98 & 0.70 & - \\
\hline $25 / 26$ Oct 96 & 0382.624 & 07103 & 150 & -2.6 & 2.0 & +3 & 2.0 & 10.0 & 2.0 & 10.04 & 0.68 & - \\
\hline $26 / 27$ Oct 96 & 0383.536 & 07179 & 150 & -7.6 & 1.5 & +17 & 2.0 & 2.6 & 0.3 & 10.13 & 0.66 & - \\
\hline $26 / 27$ Oct 96 & 0383.583 & 07183 & 180 & -9.4 & 1.5 & +17 & 1.0 & 2.8 & 0.3 & 10.11 & 0.65 & - \\
\hline $27 / 28$ Oct 96 & 0384.568 & 07255 & 150 & +2.5 & 1.5 & -3 & 1.0 & 1.5 & 0.2 & 10.02 & 0.61 & - \\
\hline $27 / 28$ Oct 96 & 0384.617 & 07259 & 150 & +3.6 & 1.5 & -5 & 1.0 & 1.7 & 0.2 & 10.16 & 0.62 & - \\
\hline $28 / 29$ Oct 96 & 0385.580 & 07323 & 220 & +6.4 & 2.5 & -2 & 3.0 & 2.5 & 0.3 & 10.04 & 0.63 & - \\
\hline $28 / 29$ Oct 96 & 0385.652 & 07329 & 180 & +6.0 & 2.5 & -4 & 4.0 & 3.5 & 0.6 & 10.16 & 0.61 & - \\
\hline $29 / 30$ Oct 96 & 0386.576 & 07388 & 200 & +0.1 & 2.1 & +26 & 2.0 & 1.5 & 0.1 & 9.95 & 0.45 & - \\
\hline $29 / 30$ Oct 96 & 0386.638 & 07392 & 170 & -1.2 & 2.1 & +28 & 2.0 & 1.2 & 0.2 & 9.96 & 0.46 & - \\
\hline $30 / 31$ Oct 96 & 0387.721 & 07455 & 100 & +9.1 & 3.0 & +5 & 3.0 & 2.7 & 0.6 & 9.88 & 0.52 & - \\
\hline 31/01 Nov 96 & 0388.511 & 07491 & 230 & -3.9 & 3.0 & +8 & 4.0 & 1.3 & 0.2 & 10.04 & 0.73 & - \\
\hline $31 / 01$ Nov 96 & 0388.573 & 07495 & 200 & -5.3 & 3.0 & +13 & 3.0 & 1.7 & 0.2 & 9.99 & 0.75 & - \\
\hline 17/18 Aug 97 & 0678.743 & 09972 & 52 & +9.0 & 3.0 & +1 & 1.0 & 5.2 & 0.8 & - & - & - \\
\hline 19/20 Aug 97 & 0680.719 & 10168 & 40 & 0.0 & 2.0 & - & - & 1.1 & 0.2 & - & - & - \\
\hline 19/21 Aug 97 & 0681.713 & 10258 & 50 & 0.0 & 3.0 & +14 & 1.0 & 2.3 & 0.4 & — & - & - \\
\hline 21/22 Aug 97 & 0682.719 & 10344 & 40 & -1.0 & 2.0 & - & - & 0.7 & 0.1 & - & - & - \\
\hline $04 / 05$ Nov 98 & 1122.554 & 16418 & 60 & +7.0 & 2.0 & +6 & 3.0 & 2.6 & 0.4 & 10.69 & - & - \\
\hline 04/05 Nov 98 & 1122.741 & 16448 & 80 & +5.0 & 2.0 & +10 & 3.0 & 2.6 & 0.5 & - & - & - \\
\hline 05/06 Nov 98 & 1123.545 & 16652 & 70 & -6.3 & 2.1 & +18 & 5.0 & 1.6 & 0.2 & 10.67 & 0.57 & -0.44 \\
\hline 05/06 Nov 98 & 1123.622 & 16666 & 60 & -6.3 & 2.1 & +19 & 5.0 & 1.0 & 0.1 & - & - & - \\
\hline 06/07 Nov 98 & 1124.543 & 16756 & 100 & +3.5 & 2.6 & -13 & 3.0 & 1.0 & 0.1 & 10.58 & 0.60 & -0.48 \\
\hline 06/07 Nov 98 & 1124.725 & 16784 & 100 & +8.2 & 2.6 & -12 & 2.0 & 1.6 & 0.1 & - & - & - \\
\hline 07/08 Nov 98 & 1125.545 & 16907 & 80 & +0.8 & 3.0 & +12 & 1.5 & 3.6 & 0.5 & 10.66 & 0.71 & -0.10 \\
\hline 08/09 Nov 98 & 1126.657 & 17060 & 55 & -6.7 & 3.0 & +10 & 5.0 & 2.6 & 0.2 & 10.44 & 0.70 & -0.16 \\
\hline 08/09 Nov 98 & 1126.712 & 17076 & 40 & -9.2 & 3.0 & +11 & 3.0 & 2.3 & 0.2 & - & - & - \\
\hline 09/10 Nov 98 & 1127.546 & 17161 & 50 & +2.0 & 2.0 & -3 & 3.0 & 1.4 & 0.2 & 10.17 & 0.76 & - \\
\hline 09/10 Nov 98 & 1127.718 & 17189 & 60 & +6.9 & 3.0 & -3 & 2.0 & 2.7 & 0.3 & - & - & - \\
\hline $19 / 20$ Oct 99 & 1471.754 & 22344 & 80 & +6.4 & 1.4 & +8 & 1.0 & 3.9 & 0.8 & 10.29 & 0.76 & - \\
\hline $20 / 21$ Oct 99 & 1472.758 & 22506 & 70 & -9.9 & 1.2 & +24 & 2.0 & 2.7 & 0.4 & 10.45 & 0.67 & - \\
\hline $21 / 22$ Oct 99 & 1473.723 & 22649 & 60 & +2.9 & 2.2 & +5 & 2.0 & 8.0 & 2.0 & 10.63 & 0.67 & 一 \\
\hline $23 / 24$ Oct 99 & 1475.753 & 22838 & 50 & -0.8 & 2.6 & +9 & 5.0 & 1.5 & 0.2 & 10.98 & 0.64 & - \\
\hline 25/27 Nov 99 & 1509.633 & 23018 & 60 & -5.5 & 1.6 & +20 & 2.0 & 1.1 & 0.2 & 10.50 & 0.62 & 一 \\
\hline 27/28 Nov 99 & 1510.709 & 23212 & 120 & +2.4 & 1.2 & +8 & 1.0 & 2.6 & 0.3 & 10.20 & 0.63 & - \\
\hline
\end{tabular}

the possible contribution from RW Aur BC in the emission lines was less than 1 percent.

The 43 spectral orders registered in one CCD frame cover a region of 3500 to $11000 \AA$ with a length of $140 \AA$ per order near $\mathrm{H} \alpha$. Usually, we took two exposures of 20 min which were added into one spectrum; this gave a useful range of 3900 to $9000 \AA$ with some gaps in the red. In 1996, we took spectra with a shift in the échelle position, so that the whole spectral range was covered without gaps. In some nights, two spectra were taken with an interval of a few hours.

The CCD images of the échelle spectra were obtained and reduced with the $4 \mathrm{~A}$ software package (Ilyin 2000). The standard procedure involves bias subtraction, esti- mation of the variances of the pixel intensities, correction for the master flat field, scattered light subtraction with the aid of 2D-smoothing splines, definition of the spectral orders, and weighted integration of the intensity together with elimination of cosmic spikes.

The wavelength calibration was done with a ThAr comparison spectrum; one was taken before and one after each individual object exposure to eliminate any temporal changes in the spectrograph during the exposure. The wavelength solution incorporates the Gaussian-centred positions, wavelengths, and times of all detected spectral lines from the two comparison images. The wavelength for every pixel in the stellar spectrum is calculated for the time of its mid-exposure. The wavelength solution also 


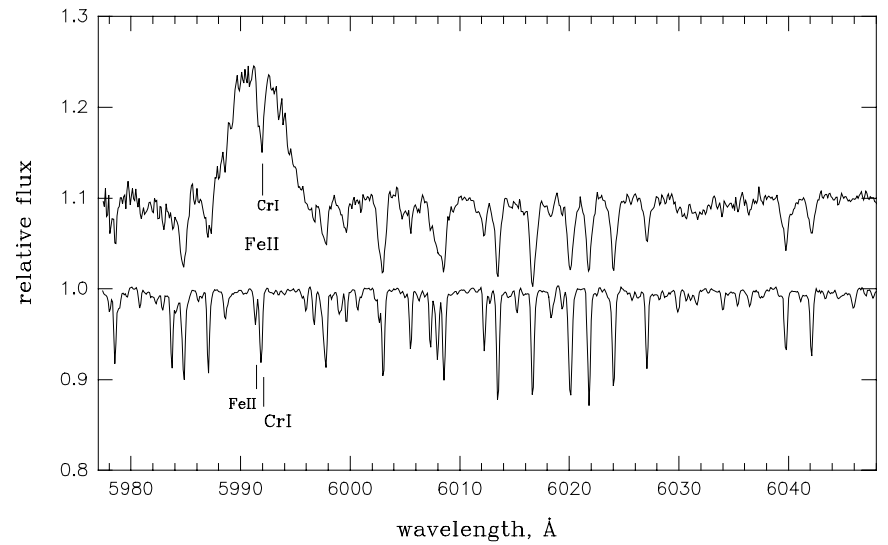

Fig. 3. Upper: the spectrum of RW Aur A (average of 1998). Note that the broad emission belongs to Fe II $5991.37 \AA$, and the superimposed narrow absorption to Cr I $5991.86 \AA$. Lower: the spectrum of $\gamma$ Cep (K1 III-IV), artificially veiled by a factor of 3

incorporates the positions of all detected telluric lines in the stellar spectrum which eliminates the wavelength shifts caused by the slit effect of the spectrograph. A typical error of the wavelength scale in the centre of the image is about $50-100 \mathrm{~m} \mathrm{~s}^{-1}$. The correction of the spectra for the vignetting function and for the Earth orbital motion constitute the next steps of the data reduction. Finally, the continuum was determined by fitting a smooth curve to the ratio of each individual and the mean spectrum. The spectra are transformed into the stellar restframe, i.e. the average radial velocity of $+16 \mathrm{kms}^{-1}$ is subtracted; all velocities given in this paper are in the stellar restframe.

Photometric observations were carried out with the stand-by CCD camera at NOT in $B$ and $V$. In 1996, CCD exposures were taken before and after each spectral exposures. In 1998 and 1999, the CCD photometry was done only before the spectral exposures. In addition, in 1998, $U B V$ photoelectric photometry was carried out with the Swedish $0.6 \mathrm{~m}$ telescope on La Palma. A few spectra taken occasionally in 1995 and 1997 were not accompanied by photometry.

The log of the observations is given in Table 1 together with some parameters of the spectra discussed in the following sections.

The colour-magnitude diagram in Fig. 1, including the data published by Herbst et al. (1994), covers over 30 years of observations. It shows that during our observations RW Aur A varied in brightness within one magnitude in $V$. The light-curves are shown in Fig. 2.

\section{Multicomponent structure of the spectrum}

In this section, we give a brief overview of different spectral features. Several components can be distinguished in the spectrum. The weak absorption lines (WALs) can be identified as the highly veiled photospheric spectrum of a $\mathrm{K} 1-\mathrm{K} 4 \mathrm{~V}$ star (average veiling $=3$; see Fig. 3 ). In the spectral classification, we try to avoid the lines originat-

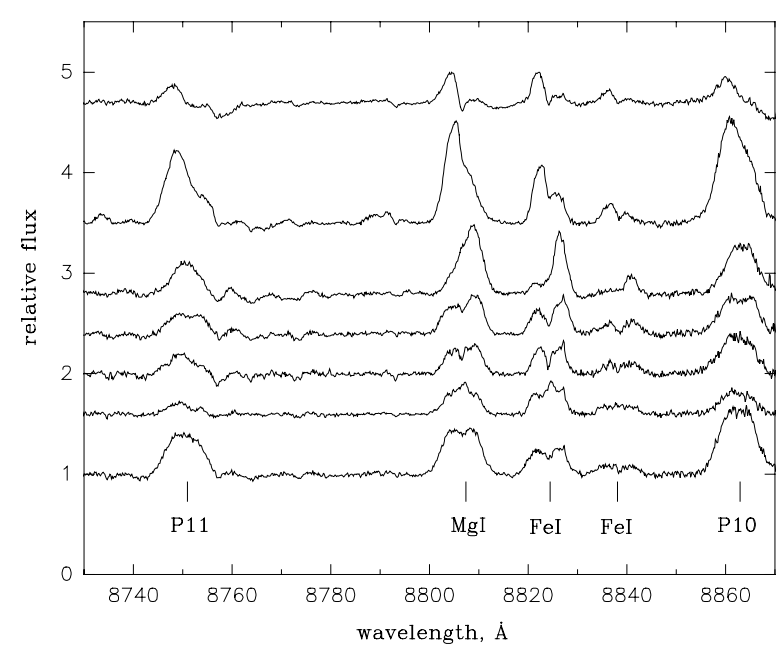

Fig. 4. An example of night-to-night variability of the broad emission lines in 1998

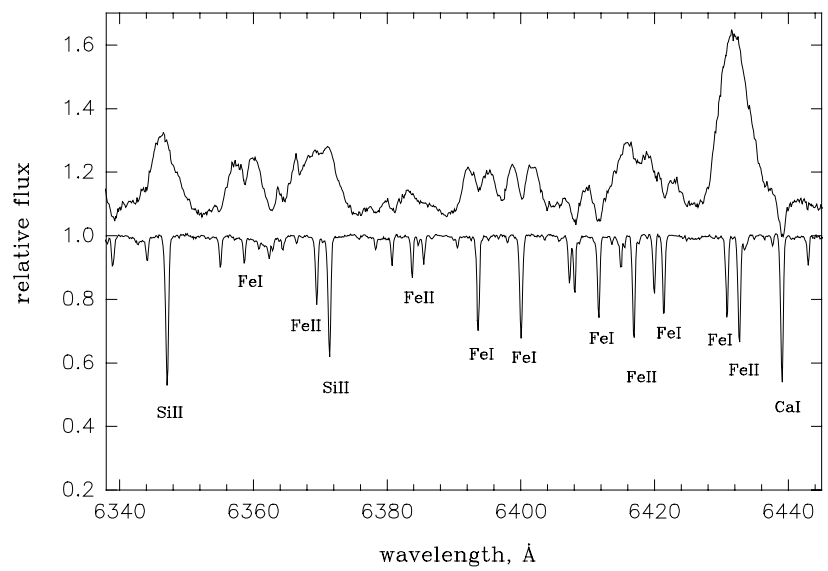

Fig. 5. A fragment of the average spectrum of RW Aur A (over all seasons) with broad emission lines compared to the spectrum of 41 Cyg (F5 II). Note that the Fe I emissions are split by narrow absorptions, while the Fe II, Si II emissions are not
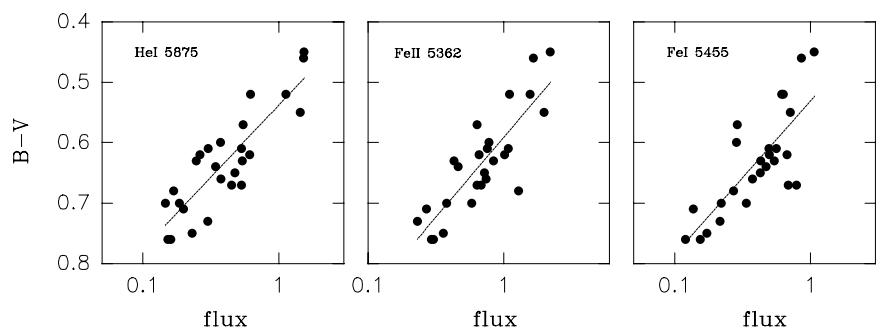

Fig. 6. Fluxes of the broad emission lines correlate with colour (fluxes in units of $10^{-12} \mathrm{erg} \mathrm{cm}^{-2} \AA^{-1}$ )

ing from low excitation levels, because they can be enhanced by additional absorption in the accreting gas well above the photospheric level (Stout-Batalha et al. 2000). This effect is quite strong in density sensitive lines, like Ba II $6141 \AA\left(\chi_{\text {exc }}=0.7 \mathrm{eV}\right)$. More details about the accretion enhancement of absorption lines, or shell lines, are given in Sect. 7. The width of the WALs, if interpreted only as rotationally broadened, corresponds on average to $v \sin i=30 \mathrm{~km} \mathrm{~s}^{-1}$, but was found variable from night to 

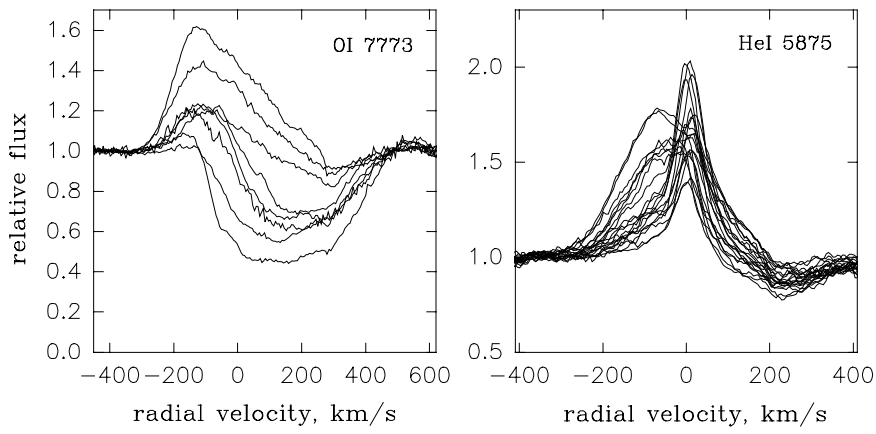

Fig. 7. Variability of the inverse P Cyg-profiles of O I $7773 \AA$ and He I $5875 \AA$

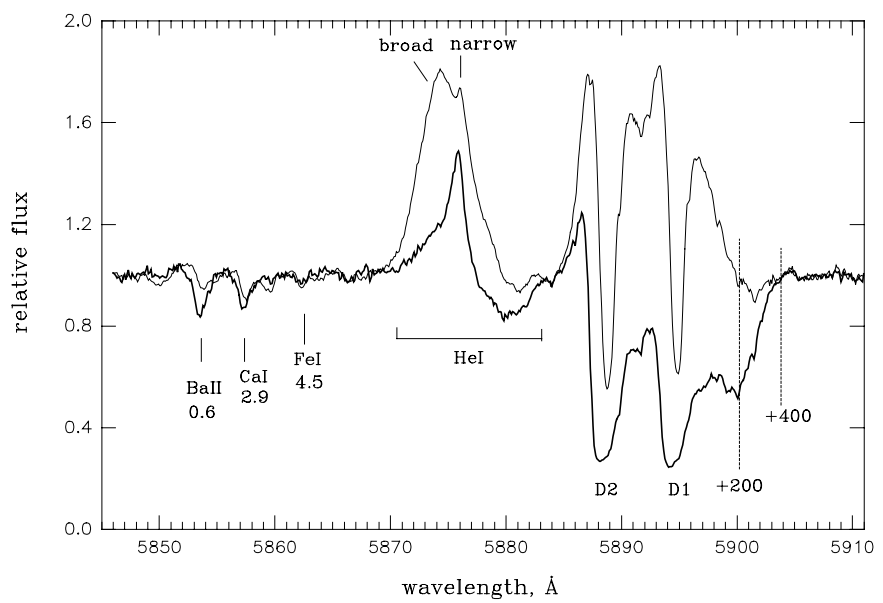

Fig. 8. The two most different spectra show the range of variability in the region of the $\mathrm{NaI} \mathrm{D}$ and He I lines. Note the strengthening of the BaII absorption when the accretion components are stronger. The numbers at the blue lines are the excitation potentials of the lower levels in $\mathrm{eV}$; those at the $\mathrm{Na}$ I lines indicate velocities relative to D1

night from 16 to $40 \mathrm{~km} \mathrm{~s}^{-1}$. Variability in radial velocity of the WALs is discussed in Sect. 6. Hereafter, we refer to these weak absorption lines as the "photospheric spectrum", although there may be a contribution from the layers above the photosphere (the shell). The spectral region, where the photospheric spectrum is least blended with emission lines, is shown in Fig. 3. In some nights, the veiling was so high that very little of the photospheric spectrum remained visible.

An outstanding characteristic of the spectrum are the numerous intensive broad emission lines (BELs), most of them belonging to neutral and singly ionised metals. The FWHM of the BELs is $200-280 \mathrm{~km} \mathrm{~s}^{-1}$ for FeI and Fe II lines, and up to $500 \mathrm{~km} \mathrm{~s}^{-1}$ for $\mathrm{H} \alpha$. The line profiles of the BELs are variable on a time scale of one day. No large variations of the line profiles were noticed during 1-2 hours. An example of the BELs' variability is shown in Fig. 4. The blue and red wings of the BELs at the line base remain symmetrically extended to $\pm 200-250 \mathrm{~km} \mathrm{~s}^{-1}$, while the intensities of the red and blue parts of the profile can change considerably.

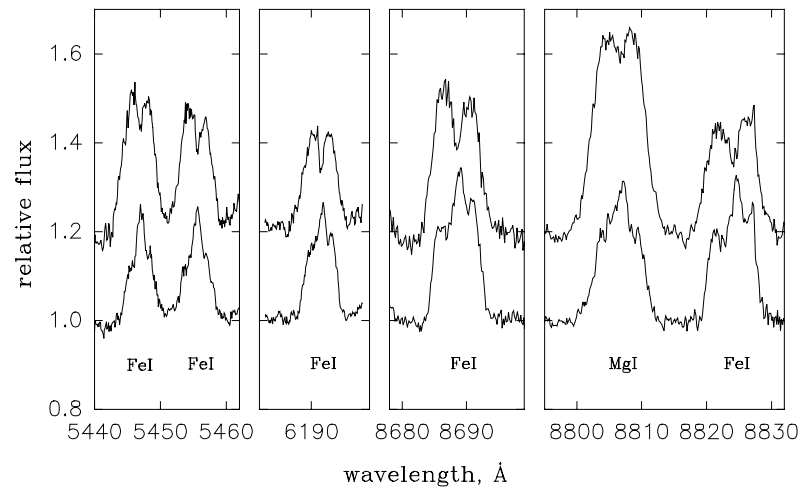

Fig. 9. The narrow absorptions on top of the broad emissions (upper spectra) turn into narrow emissions (lower spectra) when the veiling is very high

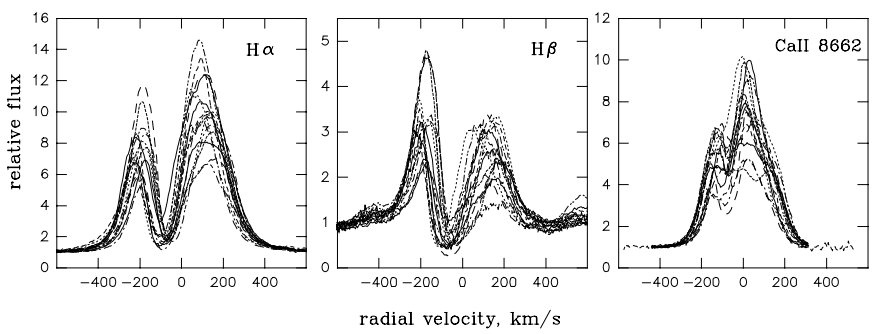

Fig. 10. Variability of lines with blue-shifted central absorption components

Most of the BELs can easily be identified using the spectrum of the supergiant 41 Cyg (F5 II) for comparison (see Fig. 5). Note, that narrow absorptions, similar to the WALs, can be found superimposed on top of the broad Fe I emissions, but are usually absent in the Fe II emissions. As a result, the BELs of Fe II look split into two parts, while the BELs of FeII and other ions have a more triangular profile. These narrow absorptions on top of emission lines vary in radial velocity and width in correlation with the WALs. One might identify them as the photospheric lines seen through the optically thin emission. Then, the absence of these absorptions on top of the Fe II lines is understandable: the Fe II lines are very weak in the photospheric spectrum of a $\mathrm{K}$ dwarf. However, the average profile of these absorptions is systematically broader than that of the WALs.

We measured the equivalent widths of 25 almost unblended, broad emissions of Fe I and Fe II observed in the spectrum with the most intensive lines, and in the spectrum with the least intensive lines. Then, the equivalent widths were converted into fluxes using our photometric data. In both cases the lines can be brought to a curve of growth with $T_{\mathrm{e}}=4300 \pm 500 \mathrm{~K}$ and $\log n_{\mathrm{e}}=9 \pm 0.5$. However, the lines can be formed in non-LTE conditions, and these values should be considered as rough estimates.

The fluxes in the emission lines show no clear correlation with the brightness of the star. Instead, there is a good correlation between the line fluxes and the $B-V$ colour (Fig. 6). This correlation is partly due to the contribution of the emission lines to the $B$ and $V$ magnitudes. 
For the spectrum with the most intensive emissions, we estimated the contribution to the $B$ passband as 0.4 , and to the $V$ passband as 0.2 . That is, most of the $B-V$ range in Fig. 6 is caused by this effect of the emission lines. The full range of variations in $B-V$ is, however, much larger (see Fig. 1). Other mechanisms, like temperature variations or extinction by circumstellar dust may enter.

The next obvious spectral features are the red-shifted absorption components in many lines. We will refer to these as the accretion components. In some lines, like the O I $7773 \AA$ triplet, Na I $D_{1} / D_{2}$, He I $D_{3}$, the accretion components are present permanently, though strongly variable in strength. The maximum velocity (extension of the red wing) is about $400 \mathrm{~km} \mathrm{~s}^{-1}$. Examples of these variations are shown in Fig. 7. Note, that the residual intensity at the bottom of the red-shifted absorption in the oxygen line can be as small as 0.4 of the continuum intensity. In Fig. 8 we show the two most differing spectra in the region of the $\mathrm{D}_{1}, \mathrm{D}_{2}$ and $\mathrm{D}_{3}$ lines. In the following analysis we will use as an "accretion parameter" the equivalent width (EW) of the $\mathrm{D}_{1}$ absorption between the velocities of +200 and $+400 \mathrm{kms}^{-1}$. There are many other spectral lines, both neutrals and ions, which occasionally show strong accretion components. The spectrum of the accreting gas is described in more detail in Sect. 7. Because of the accretion components, the maximum intensity (or centre of gravity) of the BELs is usually blue-shifted. For example, for He I broad: $-36 \mathrm{kms}^{-1}$; Fe II $5316 \AA$ : $-28 \mathrm{kms}^{-1}$; Pa 13: $-25 \mathrm{~km} \mathrm{~s}^{-1}$; Fe I $5455 \AA$ : $-14 \mathrm{~km} \mathrm{~s}^{-1}$ and Fe I $6191 \AA$ : $-8 \mathrm{~km} \mathrm{~s}^{-1}$

Besides the BELs, there are a few narrow emission lines (NELs) with FWHM of about $40 \mathrm{~km} \mathrm{~s}^{-1}$. A comprehensive study of the narrow emission lines in spectra of $\mathrm{T}$ Tauri stars (including RW Aur) was done by Batalha et al. (1996) with one conclusion being that the lines are formed near the magnetic footpoints of the accretion column. In our spectra, the narrow emission components are clearly visible in He I $5875 \AA, 6678 \AA$ and $7065 \AA$. These lines have both broad and narrow emission components. The line profile of $\mathrm{He}$ I can be decomposed into three Gaussians: a broad emission with FWHM $=200-250 \mathrm{~km} \mathrm{~s}^{-1}$, centred at $-40 \ldots-50 \mathrm{~km} \mathrm{~s}^{-1}$, a narrow emission with FWHM $=35-60 \mathrm{~km} \mathrm{~s}^{-1}$, centred at about $+10 \mathrm{~km} \mathrm{~s}^{-1}$, and an accretion component with FWHM $=150 \mathrm{~km} \mathrm{~s}^{-1}$, centred at about $+250 \mathrm{~km} \mathrm{~s}^{-1}$. Only a narrow component is present in the He II $4686 \AA$ emission, at the average radial velocity of $+20 \mathrm{kms}^{-1}$. Occasionally, weak narrow peaks can be found on top of many other lines in the spectrum which shows the highest veiling (HJD 2450382.5), as shown in Fig. 9. The line $\mathrm{D}_{3}$ is present in all of our spectra (it falls in the middle of the spectral order), and therefore we use it for the analysis of the NEL correlation with other parameters in Sect. 6 .

A blue-shifted absorption component indicating gas outflow (wind) is a typical characteristic of $\mathrm{H} \alpha, \mathrm{H} \beta, \mathrm{Na}$ I D and the IR triplet of Ca II. Examples of variability in these line profiles are shown in Fig. 10.
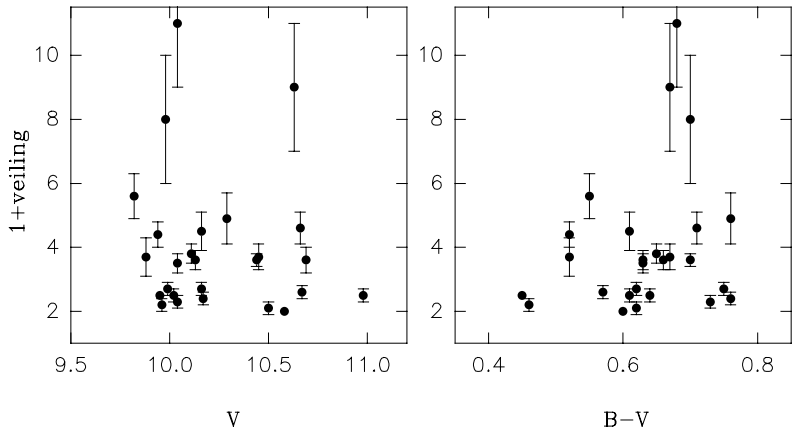

Fig. 11. Veiling versus brightness and colour

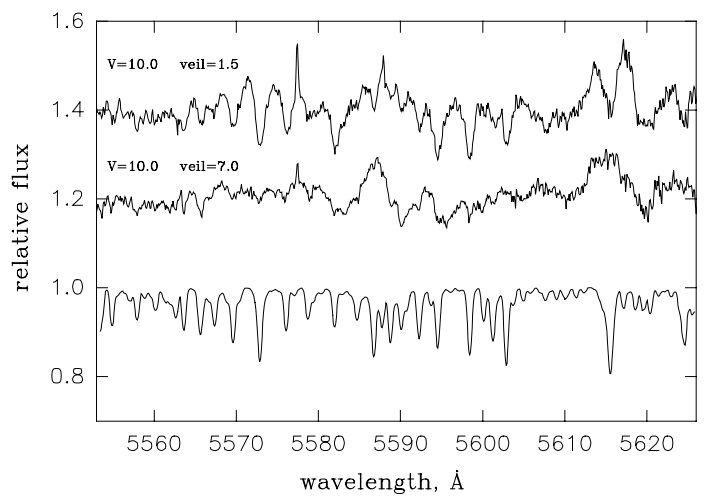

Fig. 12. Two spectra with very different veiling, but with the same brightness of the star. Lower: the spectrum of $\gamma$ Cep artificially veiled by a factor 2

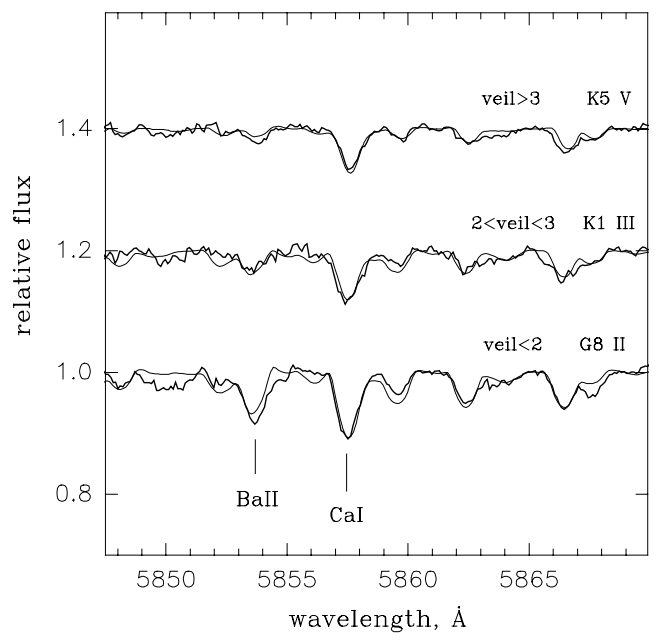

Fig. 13. Solid lines: average spectra of RW Aur A at different levels of veiling. Thin lines: comparison spectra of $40 \mathrm{Peg}$ (G8 II), $\gamma$ Cep (K1 III-IV) and 61 Cyg A (K5 V), artificially spun up to $v \sin i=30 \mathrm{~km} \mathrm{~s}^{-1}$ and veiled to the same levels as RW Aur A

And, finally, forbidden lines are always present in the spectra, e.g. [O I] $6300 \AA$, [S II] $6716 \AA$ and $6731 \AA$. The line profiles are similar to those published by Hamann (1984).

\section{Veiling versus brightness}

The veiling of the photospheric spectrum was determined in several spectral regions: $5556-5610 \AA$ (Fe I, Ca I), 
5719-5751 $\AA$ (V I, Fe I), 5855-5870 (Ca I), 6010-6045 (Mn I, Fe I). In the blue region of the spectrum, the broad emission lines are blended, and there are no continuum windows. Therefore we could not select photospheric lines useful for veiling determinations shortward of $5000 \AA$.

The veiling was derived using two different methods. The first one is based on the method described by Hartigan et al. (1989). This method consists of a comparison, within a small wavelength band of a few tens of $\AA$, between the spectra of the T Tauri star with that of a template star. The latter is chosen so that its spectrum accurately matches the unveiled photospheric spectrum of the target. The code implemented in Porto also provides the radial velocity and the rotational broadening of the target star when compared with the template star. Normally the code adjusts simultaneously four parameters: the radial velocity, $v \sin i$ and two parameters related to the continuum excess. The code also provides mechanisms for constraints on parameter values, useful when some free parameters have been measured previously using a different method.

For RW Aur A we used $\gamma$ Cep (K1 III-IV) and $61 \mathrm{Cyg}$ A (K5 V) as template stars. Both templates give similar results within the uncertainties.

The second method is based on measurements of the equivalent widths of selected absorption lines in spectra of the target and the template star: veiling $=$ $\mathrm{EW}$ (template)/EW(TTS)-1. The template spectrum was rotationally broadened to $v \sin i=25 \mathrm{~km} \mathrm{~s}^{-1}$ in order to get the same blends of lines as in the spectrum of RW Aur A. In this method we assume that the line width $(v \sin i)$ is the same in all the spectra, and that the EW of a line varies due to the veiling variations. The errors come mainly from the uncertainty in the continuum level, especially in the spectra with stronger emission lines.

There is a correlation in the time variation of the veiling derived from different spectral regions. However, the absolute value of the veiling can differ significantly from region to region. In Table 1 we give the average values of the veiling obtained by the two methods derived from the Fe I lines in the region of 5556-5610 $\AA$. These are the strongest lines of higher excitation potentials, and they are less sensitive to temperature, which makes the selection of the template less critical.

The most unexpected result of this study is that no correlation was found between the veiling and the brightness of the star, although the brightness varied by one magnitude, and the veiling varied over a wide range (Fig. 11). Moreover, quite different veiling factors were found in spectra taken at the same brightness of the star, for example, in the nights HJD 2451124 and 1125, when photometry was made at two telescopes. Another example (HJD 2450382 and 0384) is given in Fig. 12 showing different veilings (1.5 and 7.0) but no significant difference in brightness. The veiling was determined in the spectral region of the $V$ band. If the veiling were caused by additional continuum radiation in this spectral region, the brightness difference in $V$ would be more than one magnitude!

Another oddity is the variability of " $v \sin i$ " from night to night in the range 16 to $40 \mathrm{~km} \mathrm{~s}^{-1}$. The effect is real, not instrumental, e.g. the sky line at $5577 \AA$ remains perfectly narrow in the spectra which show broadened photospheric lines. The minimal value of $v \sin i$ of $16 \mathrm{~km} \mathrm{~s}^{-1}$ can be considered as due to stellar rotation. No periodicity in the variations of the " $v \sin i$ " parameter was found. We suggest that the broader lines are contaminated by an additional absorption from layers above the photosphere. An argument in favour of this suggestion can be seen in the variability of the BaII $5853 \AA$ line. The line was enhanced in some nights, that is the ratios of BaII/Ca I or $\mathrm{Ba}$ II/Fe I were too large for a dwarf. This strong line, indicating $\log g \leq 2$ like in supergiants, was observed mostly in spectra with low veiling (Fig. 13).

We conclude that Ba II is formed in a shell above the photosphere. The same shell must contribute also to absorption in many other lines, which make them deeper and broader, thus simulating low veiling and variable $v \sin i$. The spectral features of a "warm shell" in RW Aur were also found by Herbig \& Soderblom (1980). More information about the shell lines is given in Sect. 7 .

Hence, we conclude that the variations of the veiling we observed in RW Aur A are caused by at least two different processes: 1) the "true" veiling due to continuum + line emission, and 2) absorption in the shell which imitates low veiling. This may partly explain why the observed "veiling" is not correlated with brightness, although the considerable increase in veiling at constant brightness remains a mystery.

\section{Extinction and stellar parameters}

Most of the time RW Aur A is probably seen through foreground circumstellar dust, and it is difficult to derive any precise values of the interstellar extinction to the star. We have integrated all FUV spectra obtained with the International Ultraviolet Explorer (IUE) of RW Aur A covering the spectral region with the interstellar $2200 \AA$ signature. The integrated spectrum shows a number of blended emission lines, and we can only set a rather high upper limit of the average extinction of $A_{V} \leq 0$. 7 . Ghez et al. (1997) give $A_{V}=0.3$ as a possible upper limit. We have taken one spectrum of RW Aur B, 1". 4 from A, showing only weak traces of interstellar Na D absorption. The total equivalent width of these lines amount to $0.2 \AA$, also consistent with a low interstellar extinction to RW Aur. Hence, we assume that the interstellar extinction to RW Aur is low, which is consistent with its location outside any molecular cloud boundary.

When the star is brightest and bluest, we expect the circumstellar extinction to be minimal. A minimum of $B-V=0.45$ was observed on HJD 2450386 (see Table 1). The colour is too blue for a $\mathrm{K}$ dwarf, indicating the presence of a hotter continuum source also responsible for the veiling. On this occasion, the star had 


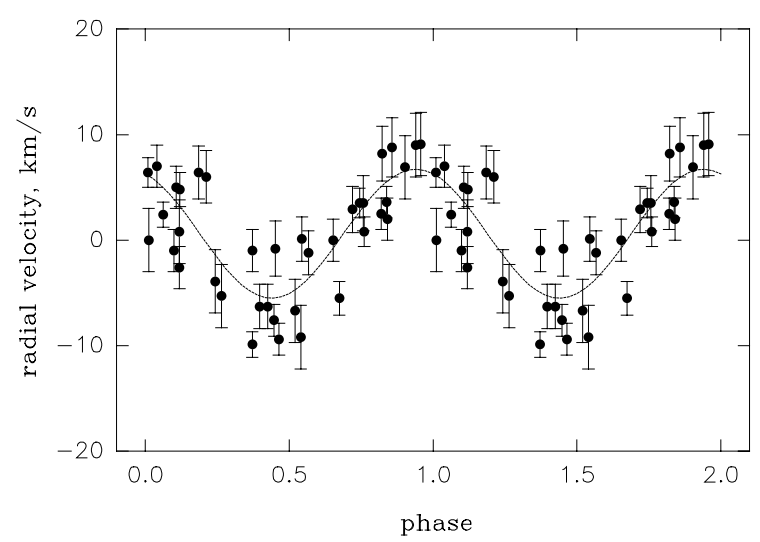

Fig. 14. Phase diagram of the radial velocity variations of the weak absorption lines, $P=2$ d. 7721

$V=9 \mathrm{~m} 95$ and veiling $=1.5$. Taking into account the correction for the contribution of emission lines $(0.2$ in $V)$ and the correction for the veiling $(2.5 \log (1+$ veiling $))$, we find a corrected $V=11$. 14 . With the distance of $140 \mathrm{pc}$ (Elias 1978) and $A_{V}=0 \cdot 3$, the absolute magnitude is $M_{V}=5{ }^{\mathrm{m}} 11$. Using a bolometric correction appropriate for the spectral types $\mathrm{K} 1-\mathrm{K} 4$, which is $\mathrm{BC}=$ $0^{\mathrm{m}} 2 \ldots 0^{\mathrm{m}} 5$, we get $M_{\text {bol }}=4^{\mathrm{m}} \cdot{ }^{2} \ldots 4^{\mathrm{m}} 6$, which corresponds to $L / L_{\odot}=0.85 \ldots 1.1$. The derived luminosity represents a lower limit, since circumstellar extinction can be present.

From the spectral type range and the luminosity we can estimate the radius of the star as $1.3 \ldots 1.5 R_{\odot}$. Since the luminosity is a lower limit, the radius is also a lower limit.

With the lower limit of the luminosity, and with the range in spectral type, we obtain a lower limit for the mass of the star of about $1.1 M_{\odot}$ according to evolutionary model tracks (e.g. Palla \& Stahler 1999). Notice, that with these values of mass and radius, the free-fall velocity (starting from a distance of 10 stellar radii) is about $500 \mathrm{~km} \mathrm{~s}^{-1}$ at the stellar surface. This is in a good agreement with the observed maximum accretion velocity of $400 \mathrm{~km} \mathrm{~s}^{-1}$.

\section{Periodicities in spectral line variations}

In Paper I, we have reported already the discovery of periodic variations in the photospheric and emission lines. Since then we have collected more spectra in 1999; the new spectra confirm that the period and the phase remain stable. All radial velocities were measured by crosscorrelation with the template spectrum of $\gamma$ Cep (K1 IIIIV) in the regions 5550-5610 $\mathrm{A}$ and $6000-6050 \AA$. The average radial velocity derived from the photospheric spectrum is $+16 \mathrm{~km} \mathrm{~s}^{-1}$ and is subtracted from all measured heliocentric velocities. The radial velocities of the WALs vary from -6 to $+6 \mathrm{~km} \mathrm{~s}^{-1}$. A periodogram analysis indicates a period most likely in the range from 2.5 to 2.9 . There is a number of almost equal, equidistant peaks, separated in frequency by $1 /$ year. The strongest peak is at

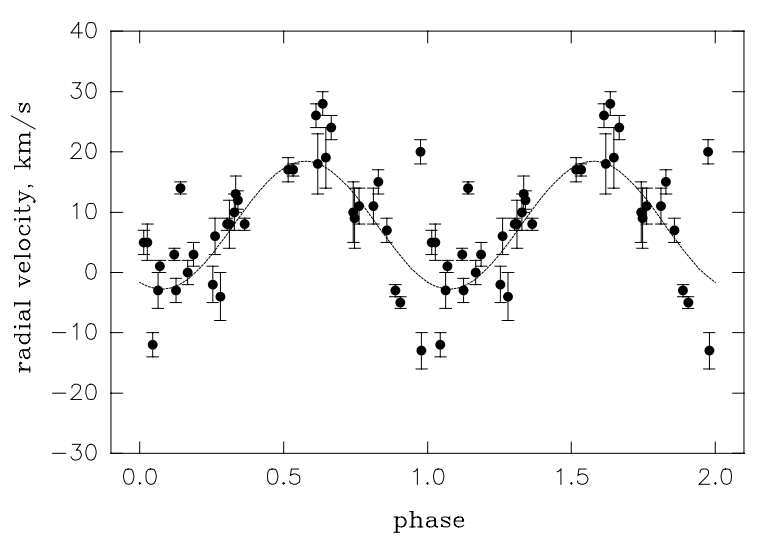

Fig. 15. Phase diagram of the radial velocity variations of the He I $5876 \AA$ narrow emission, $P=2$ d 7705

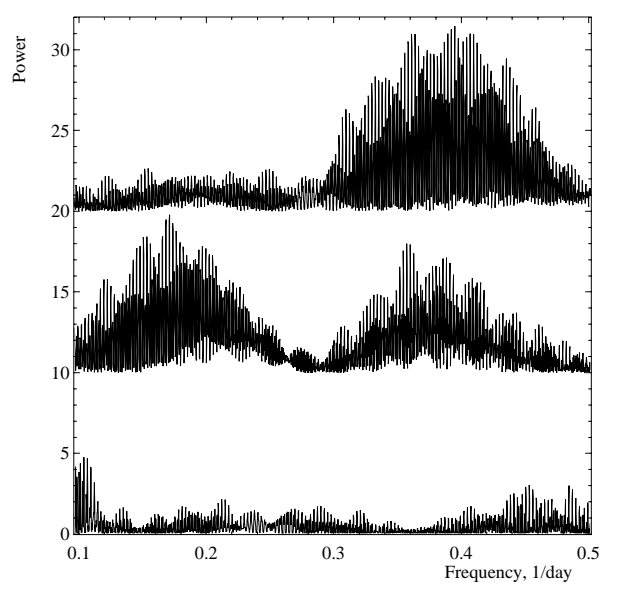

Fig. 16. Power spectra: upper - EW of the narrow emission of He I $5876 \AA$, centre - EW of the broad emission of He I $5876 \AA$, lower - spectral window
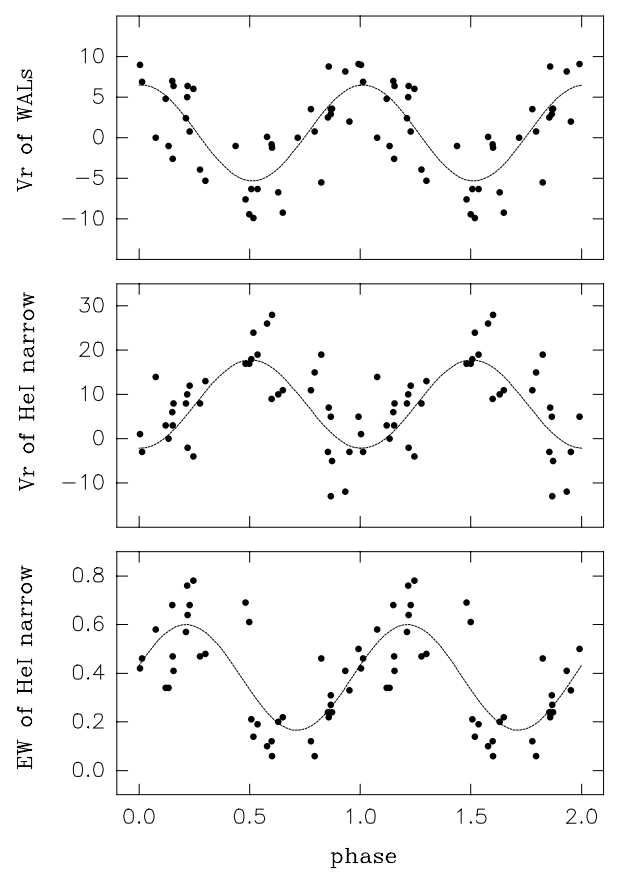

Fig. 17. Phase shifts between the radial velocities of the WALs, the radial velocities of the He I NELs and EWs of He I NELs, $P=2$ d 7710 


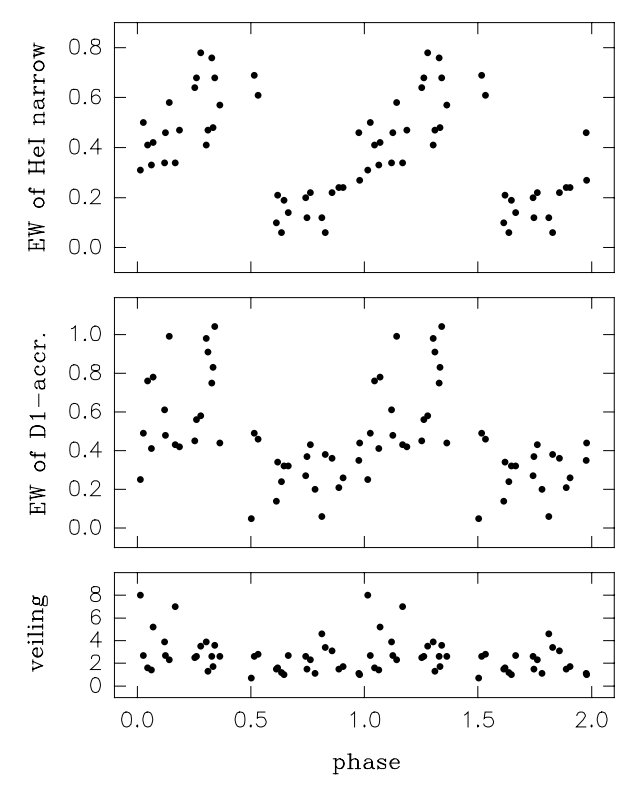

Fig. 18. Phase diagrams of the EW of the He I narrow emission (upper panel), the accretion components of $\mathrm{NaI}_{1}$ (middle panel) and the veiling (lower panel), $P=2$ d. 7705

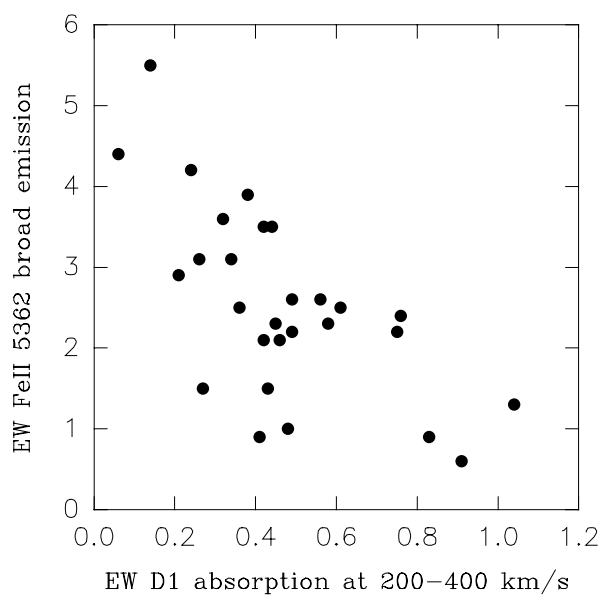

Fig. 19. Anti-correlation of the accretion components of $\mathrm{Na}_{\mathrm{I}} \mathrm{D}_{1}$ and the EW of the broad emission of Fe II $5362 \AA$

$P=2$ d7721. A phase diagram with this period is shown in Fig. 14.

In addition to the WALs, we also analyzed the periodicities in other groups of spectral features: NELs, BELs and accretion components. We found that the WALs, NELs and accretion components all show about the same set of frequencies in the power spectrum, with a period in the range of 2.5 to 2.9 . The BELs show a twice longer period in the range of 5 to 6 days. The period around 2.77 is also present in the variations of the BELs, but with less power than the $5-6$ days period. This is illustrated in Fig. 16.

With our set of data we cannot prove that there is only one and the same period in the variations of the WALs, NELs and accretion components. For example, the power spectrum of the NELs shows that the most significant peaks are slightly shifted with respect to those of the WALs. The best period for the radial velocities of the narrow He I line is $P=2$ d.7705 (compared to 2 d 7721 for the WALs). The corresponding phase diagram is shown in Fig. 15. Note the systematic shift in the mean radial velocity of the He I NEL by about $+8 \mathrm{kms}^{-1}$. The velocity amplitude is larger than that of the WALs.

The EW of the He I NEL varies from 0.15 to $0.60 \AA$ and shows the same periods as the radial velocity, with the strongest peak at $P=2$ d.7705. In order to check if the WALs and the He I emission vary in phase or with a phase shift, one must plot a phase diagram with a common period. A compromise period of $P=2$ d 7710 was used for the diagram shown in Fig. 17. The result is that the radial velocity of the WALs and the He I NEL vary in anti-phase (phase shift 0.5), while the EW of the He I NEL shows a phase shift of about 0.25 . Any other common period in the 2.5 to 2.9 interval shows the same phase shifts.

The He II $4686 \AA$ has only a narrow emission component, and varies in correlation with the narrow emission in He I $5876 \AA$. However, the mean radial velocity is even more red-shifted, $+20 \mathrm{~km} \mathrm{~s}^{-1}$.

The EWs of the accretion components vary in phase with the EW of the He I narrow emission. This is shown in Fig. 18 for the $\mathrm{Na}_{\mathrm{I}} \mathrm{D}_{1}$ red-shifted absorption between +200 and $+400 \mathrm{~km} \mathrm{~s}^{-1}$. The stronger the He I NELs, the larger is the accretion absorption.

The EWs of the broad emissions of He I, Fe I, Fe II vary in correlation with each other. An anti-correlation exists between the variations of the EWs of the accretion components and the EWs of the broad emission lines of He I, Fe I, Fe II (Fig. 19). More about correlations between different spectral features is given in the next section, where the spectral line profiles formed in the accreting gas are discussed.

No periodicity was found in the veiling variations, and there is no obvious correlation between the veiling and any other spectral feature. Only a weak correlation exists between the veiling and the EW of the He I narrow emission. Conversion of EWs into fluxes does not improve the correlation.

\section{The spectrum of the accreting gas}

The best indicators of accretion are the lines of $\mathrm{NaI} D$ and the O I $7773 \AA$ triplet. Occasionally, when accretion is enhanced, red-shifted components appear in many other lines. In one night (HJD 2450388.5) the accretion was exceptionally strong. The accretion components were present in almost all the emission lines, which gives an opportunity to describe the spectrum of the accreting gas in more detail. Profiles of selected lines of different excitation potentials are shown in Fig. 20. The common feature is that the accretion line profile consists of two different components: a low velocity absorption at about $0 \mathrm{~km} \mathrm{~s}^{-1}$ or slightly blue-shifted, and a high velocity absorption with the bottom around $+250 \mathrm{~km} \mathrm{~s}^{-1}$ and extending to $+400 \mathrm{kms}^{-1}$. The low excitation lines of KI, Li I, Ba II, Ca I and Fe I all show enhanced low-velocity absorptions 

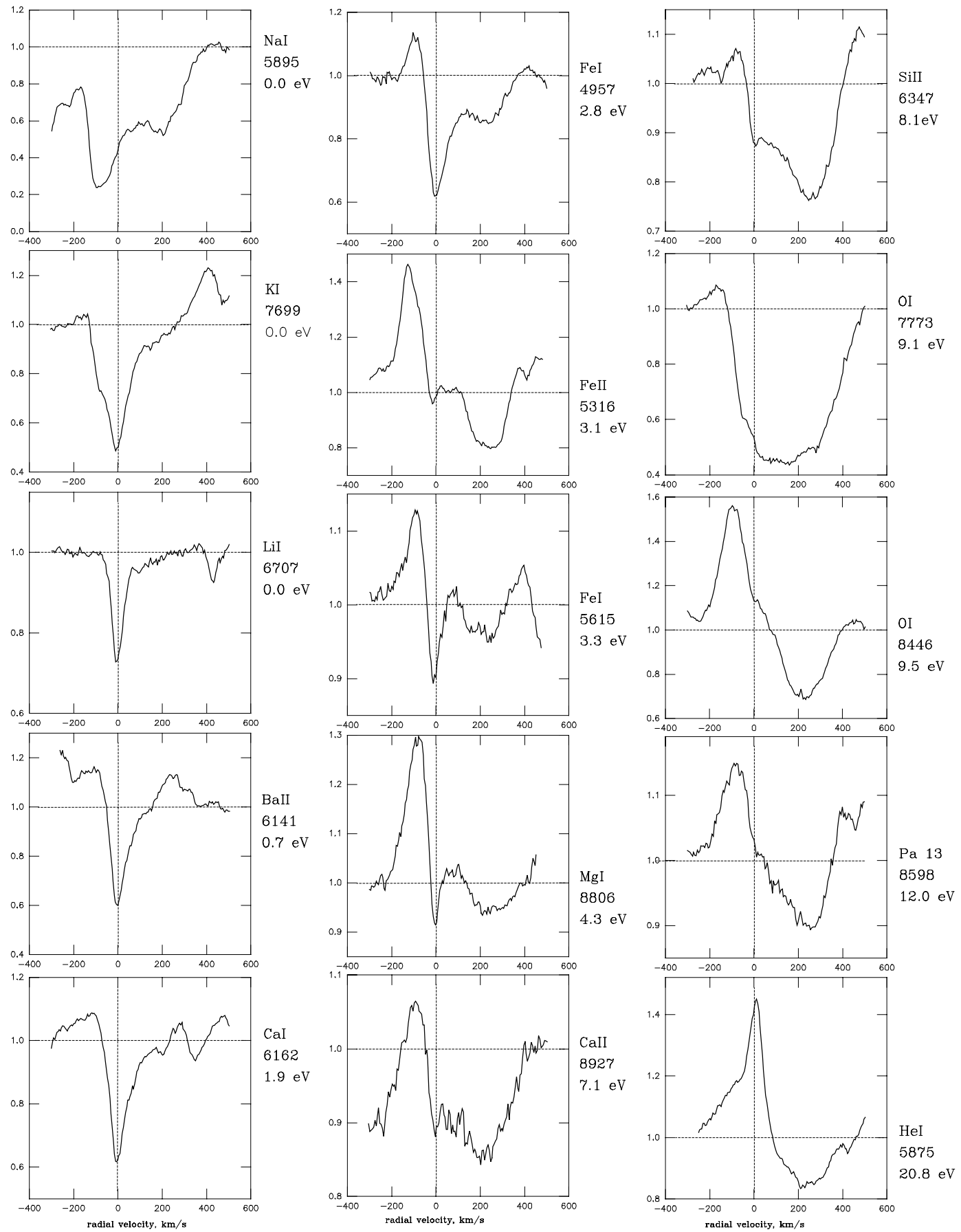

Fig. 20. Accretion components of lines of different excitation potentials in the spectrum with enhanced accretion (HJD 2450388.5)

with a weak red-extending wing. The lines of excitation potentials between 2 and $7 \mathrm{eV}$ have both low-velocity and high-velocity absorptions. The lines of the highest excitations, more than $8 \mathrm{eV}$, show mostly high-velocity components.

The presence of accretion-induced enhancements of the low excitation, low ionization lines in the spectrum of RW Aur A were reported recently by Stout-Batalha et al. (2000) with emphasis on Li I. They suggested that the enhancement of absorption at the line centre is due to additional absorption by cool gas at the beginning of the accretion flow.

The EW of the central absorption of K I $7699 \AA$ can be used as an indicator of this low velocity gas. We find a correlation between the EW of K I $7699 \AA$ and the EW of the blue-shifted absorption of $\mathrm{H} \beta$, both correlating with $B-V$ (Fig. 21). This is an observational evidence that the inflow and the outflow are related processes.

The difference between this spectrum with enhanced accretion and the average spectrum of RW Aur A is shown in Fig. 22. Note that the dips of the absorptions are 


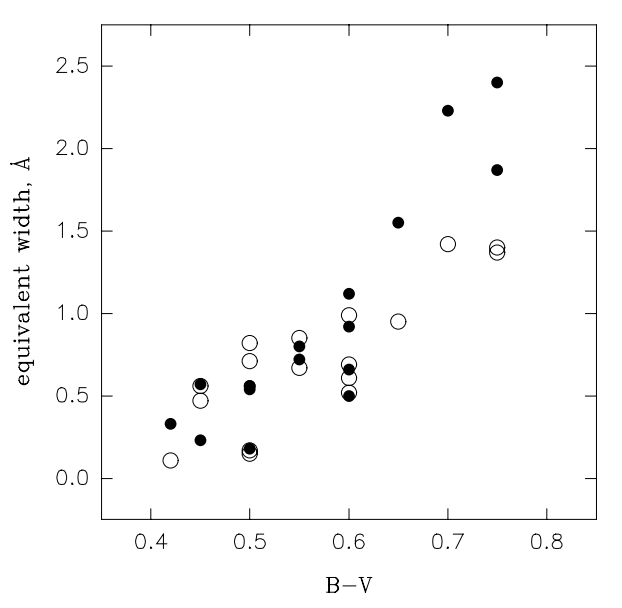

Fig. 21. Correlation of the EW of the low velocity absorption in K I $7698 \AA$ (filled dots) and of the EW of the central absorption in $\mathrm{H} \beta$ with $B-V$

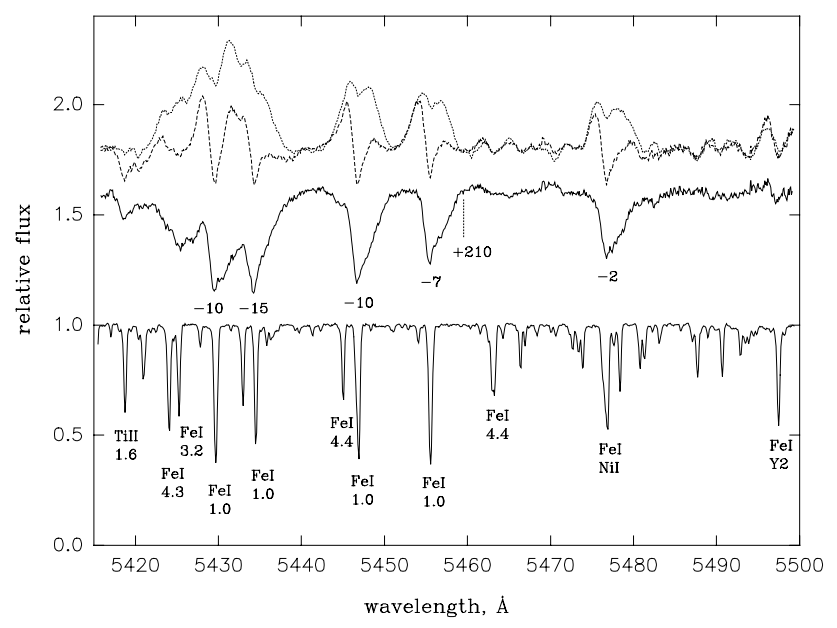

Fig. 22. The uppermost two spectra of RW Aur A are the average spectrum and the spectrum with enhanced accretion (dashed). Below them, their difference (the differential spectrum) is shown with radial velocities indicated. At the bottom we show the spectrum of $\gamma$ Cep (K1 III-IV) with line identifications and excitation potentials

blue-shifted from -2 to $-15 \mathrm{~km} \mathrm{~s}^{-1}$. The shift is larger for stronger lines (up to $-100 \mathrm{~km} \mathrm{~s}^{-1}$ in $\mathrm{Na} \mathrm{D}$ ). No molecular bands of $\mathrm{TiO}$ were found in the differential spectrum.

In several lines of neutrals and ions we measured the relative depths of the low velocity components and the relative depths at $+250 \mathrm{~km} \mathrm{~s}^{-1}$. Assuming LTE conditions we find $T_{\mathrm{e}}=4700 \pm 300 \mathrm{~K}, \log n_{\mathrm{e}}=9.2 \pm 0.6$ for the low velocity components, which are similar to the parameters obtained for the broad emission lines. The high-velocity absorptions are formed at higher temperature $(>8000 \mathrm{~K})$ and higher densities $\left(\log n_{\mathrm{e}}>13\right)$ but different sets of lines give different values.

\section{Discussion}

The extremely complex, yet regular patterns of spectral variability, together with the "wild" photometric be- haviour of RW Aur A, challenge any attempt to model the star and its surrounding. The main result is the periodic, anti-phase variations in the radial velocities of the WALs and NELs. Such a periodicity in principle can be induced either by orbital motion of an invisible, low-mass secondary (see Paper I), which also influences the gasflows around the star, or by rotational modulation of a single star with an inclined or asymmetric magnetosphere. In the following, we present two sketches of models, which we hope can form a basis for further developments, and also provide indications of future observational tests.

Both models are critically dependent on the inclination of the stellar rotation axis and a possible stellar magnetosphere. In Paper I we discussed how the radial velocity measurements of the jets at RW Aur A can be used to restrict the range of possible orientations. Recently, C. Dougados (private communication) has communicated to us a preliminary estimate of the inclination of the counter-jet of the star, based on the proper motion and radial velocity of one knot in the jet. The inferred inclination (to the line-of-sight) is $67 \pm 4$ degrees (see also Dougados et al. 2000). We adopt this value as the direction of the jet. (The gas could move faster than the knots, in which case the value is a lower limit). In addition we will assume that the jet axis is perpendicular to a circumstellar dusty disk surrounding RW Aur A, and parallel to the axis of stellar rotation.

We need to explain the following observed properties:

1. Periodic, sinusoidal variations of the radial velocities of the WALs and NELs, and, in addition, of the equivalent widths of the NELs and the accretion components, all with a period of $P=2$ d 77 ;

2. Anti-phase between the WAL and NEL radial velocities;

3. The total amplitudes of the HeI and He II NEL radial velocities are equal, but the average velocities are redshifted with respect to the average WAL velocity He II significantly more than He I;

4. A $\sim 1 / 4$ phase-shift between the variations of the equivalent widths and radial velocities of the HeI NELs;

5. Correlation between the NEL and the accretion component equivalent widths, with a burst of accretion occurring during each cycle;

6. Periodic variations of the equivalent widths of the BELs with about the double period, $\approx 5.5$;

7. Anti-correlation between the BEL and accretion component equivalent widths.

Common for both models is that the observed variabilities of the red-shifted absorptions are related to the stream(s) of gas channeled by a magnetic field to hot spot(s) on the stellar surface. Stellar activity alone cannot account for all the details: non-axisymmetric accretion is present.

The broad emission lines with the blue-shifted maximum and red-shifted absorptions are most probably formed in a global magnetosphere threaded by streams of gas flowing towards the star (Calvet 1998 and references 
therein; Muzerolle et al. 1998). The free-fall time is much less than the period of rotation, i.e. local fluctuations in the accretion flows would result in irregular variations of the blue and red wings in the broad emission line profiles.

In projection onto the star, along the same line of sight, we see the low velocity, low temperature gas at the beginning of the accretion column, and the high velocity, high temperature gas approaching the star at the end of the accretion column (Fig. 20). The narrow emission lines of $\mathrm{He}$ can be formed within the same accretion column but very near the stellar surface, where the gas is already decelerated but still has some positive velocity. This explains the shifts in the mean radial velocity of NELs, and the correlation between the EWs of the NELs and the accretion components (Fig. 18). These narrow He I emission lines can be used for the detection of the magnetic field of this star, as was made for BP Tau by Johns-Krull et al. (1999).

The lines of Ba II discussed in Sect. 4 and the enhancements of the low excitation lines of Li I, K I, Ca I originate probably in the outer part of the magnetosphere, which acts like a shell. The strengths of these absorptions correlate with $B-V$, which may be due to the presence of dust in dense blobs of gas rising from the accretion disk. The blue-shifted "dips" in these lines indicate that a wind component is also present in the profile. Presumably, the ultraviolet blue-shifted absorption components reported by Gahm (1970) and Errico et al. (2000) originate in the same wind outside the main accretion flows.

Finally, the light variability most certainly is due to variations both in circumstellar extinction and the continuous veiling.

The properties described above are common for both models, but the origin of the periodical modulation is different.

\subsection{Binary star?}

Paper I addressed several aspects of the binary hypothesis, where it is assumed that the WAL velocity variations are due to orbital motion. First of all it is reassuring that our new observations confirm the period, amplitude and phase of the radial velocity variations of the WALs. In addition, we now have the inclination of the system (assuming that the orbital axis is aligned with the jet). We expect that the K-type primary has a mass slightly larger than $1 M_{\odot}$ (see Sect. 5). It follows that the secondary is a brown dwarf (BD) of $0.04-0.05 M_{\odot}$ in an orbit of low eccentricity at about $8-10 R_{\odot}$ from the primary (see Paper I). The orbital period of the secondary is $2 \mathrm{~d} .77$, and the centre of gravity is well inside the primary.

The basic concept of the binary star model is that the periodic spectral variability is related to the influence of a close secondary component, just like for other close binary TTSs. The HeI and He II NELs cannot originate at hot spots on the secondary unless the inclination of the system is very small (Paper I). Moreover, this hot region cannot be located between the components, for instance as the
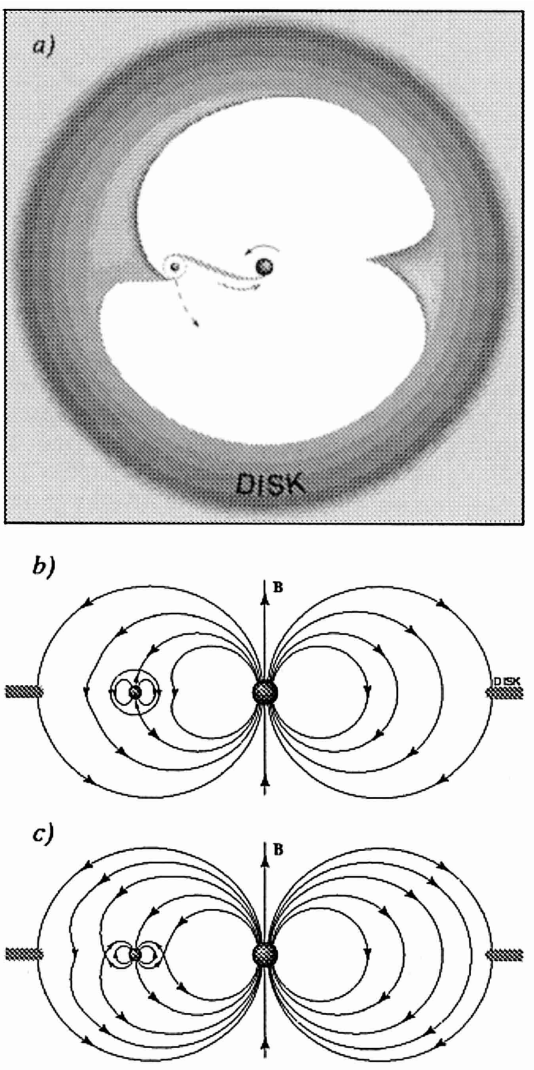

Fig. 23. In our search for mechanisms explaining how the secondary may act as a generator of enhanced accretion from an area at the disk edge to a spot on the primary we discuss gravitational perturbations leading to accretion along spirals to the secondary, where some matter is deflected and continues to fall down to a spot, possibly along a trajectory tilted relative to the primary surface (upper panel). Another concept is described in the lower two panels, showing the combined magnetic field structure of two magnetic stars. The field at the secondary looks very different if the magnetic bodies have parallel (middle panel) or anti-parallel fields (lower panel). Enhanced MHD action occurs at the disk edge closest to the secondary

result of two colliding winds, because then the amplitudes of the NEL radial velocities would be much larger than observed. However, many TTSs show narrow He I emission components thought to arise at the footpoints of global accreting flows from the disk, and we have followed this general concept.

Consequently, our discussion focusses on the following idea: global magnetospheric accretion, typical for TTSs, is present. The BELs are formed in this flow. In addition, the secondary produces enhanced accretion in one direction. This gas falls freely into regions close to the stellar surface of the primary, where deceleration and heating occurs. We have considered two possible mechanisms, which may lead to such an asymmetry of the accretion. Both these possibilities are shown schematically in Fig. 23, where the disk edge is placed at the 2:1 period resonance (i.e., the orbital period of the inner part of the disk is about 5.5 ).

One possibility is that the secondary, which has cleaned a gap in the disk, also perturbs the disk. Gas 


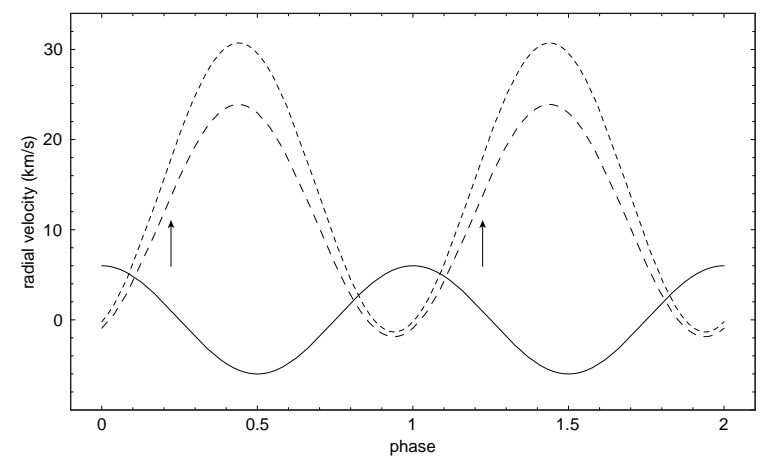

Fig. 24. Calculated radial velocity changes of the WALs (fulldrawn), the HeI NELs (dashed), and the HeII NEL (shortdashed). The phase whith expected maximum in the EWs of He are marked with arrows

spirals from the disk, preferentially to the secondary, where some matter is deflected and falls onto the primary, possibly along spiral trajectories. Similar cases have been calculated by e.g. Artymowicz \& Lubow (1996), and could explain the periodic bursts of emission observed in TTSs with close massive components in elliptic orbits, like DQ Tau (Basri et al. 1997). However, we envision a secondary in a nearly circular orbit, and it is not massive. This particular case must be followed numerically, assuming an upper limit of the ellipticity of 0.2 (according to Paper I), before any conclusions about plausibility can be made.

Another possibility is that both stars are magnetic, and that the magnetospheres interact in such a way that enhanced accretion from the disk occurs along dipole magnetic field lines to the primary in the direction to the secondary. In this case, the secondary receives rather little of the accretion flow, and acts only as a generator of enhanced MHD action in a small region of the disk edge. Carlqvist (private communication) has provided the principles for a combined magnetic field from two magnetic stars (dipoles) based on early magnetospheric models. For the configurations in Fig. 23, the surface magnetic field strength of the contracting $\mathrm{BD}$ (radius $\sim 0.3 R_{\odot}$ ) was assumed to be about $1 / 10$ of that of the primary. These two cases represent parallel and anti-parallel field orientations. Note, that for enhanced MHD action to be efficient, the disk radius must be smaller or the BD magnetic field stronger than assumed in Carlqvist's calculation. The recent discovery of a large flare on a brown dwarf by Rutledge et al. (2000) shows that the magnetic field can be strong.

We have considered several possible models for how gas is channeled into a cylinder over an area ("spot") on the primary. The narrow He I and He II lines are formed as a result of heating from an impact shock. When the secondary is closest to us, also the spot is facing us, since maximum lineflux occurs at a phase around 0.25 . The spot must also be seen all the time, and is therefore located at high latitude. A similar spot may exist on the "southern" stellar hemisphere, but is tilted out-of-view. In addition, the He lines have maximum positive velocities at phase 0.5 , implying that the final impact must occur along trajectories trailing the secondary.

Figure 24 shows one example of calculations using a simple geometrical model. In this model, the centre of the primary, the spot, and the secondary are orbiting the centre of gravity. The spot co-rotates at high latitude, where the orbital motion of the spot is small. The infall is nearly radial to the normal of the surface, but strongly tilted in longitude. In this way the anti-phase variations of the WALs and NELs can be reproduced, as well as the amplitudes of the NELs. Assuming that the EWs of He are largest at maximum projected spot area, the corresponding observed $\sim 1 / 4$ phase-shift can also be reproduced. The NELs of He I and He II are assumed to form along the same trajectory but with different average infall velocities (tracing different volumes in the cylinder). The shift in average velocity in Fig. 24 corresponds to infall velocities of $30 \mathrm{~km} \mathrm{~s}^{-1}$ (He I) and $40 \mathrm{~km} \mathrm{~s}^{-1}$ (He II).

In the binary case, part of the veiling comes from a ring around the visible pole connected to global accretion as manifested in the BELs, just as assumed for other TTSs. Superimposed is the veiling produced in the "spot". It could be that this spot is a region of enhanced accretion in the high latitude ring. Both these components can be expected to vary irregularly and independently due to variable mass inflow. Part of the veiling may also be due to narrow emission lines, as is discussed in the subsequent section.

Referring to the model descriptions above it seems worth exploring accretion paths in the combined magnetospheres of the stars. Since the disk edge is located far outside the co-rotation radius, the global field is dragged behind, in a way similar to the solar magnetic field. The field lines can therefore be expected to be curved also in longitude. The magnetosphere of the secondary acts as the motor of channelled accretion in this case.

In conclusion, it seems that the binary hypothesis can account for most of the points set above. Many of the details are presently not very well understood, and require a much deeper analysis of the physics involved. A major item is point 6 , namely the 5.5 period, which calls for an additional modulation of the BELs.

\subsection{Single star?}

Periodic variations in the accretion features are not unique to RW Aur A, and were observed also in other TTSs, like SU Aur (Giampapa et al. 1993; Petrov et al. 1996). As an explanation it was proposed that the periodicity is related to the axial rotation of the star. If the magnetic axis is not aligned with the rotational axis, the accreting gas gives rise not to hot rings, but to two elongated hot spots near the two magnetic poles of the star (Königl 1991). Then, the stellar rotation would modulate the brightness and the spectral features associated with the accretion. The same effect of the rotational modulation may appear 


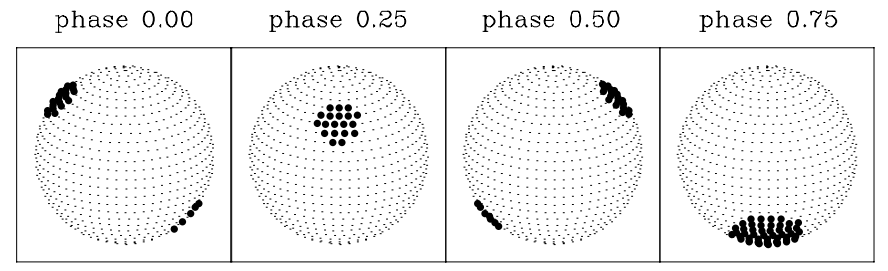

Fig. 25. A model of a star with two spots of chromospheric emission (solid dots), associated with the two footpoints of the accretion columns. The photospheric absorption spectrum contributes in each dot over the entire surface, while narrow emission lines only arise in the spot area

if the structure of the stellar magnetic field is not perfectly symmetric, with larger magnetic loops at certain longitudes. In both cases, we have non-axisymmetric magnetospheric accretion. Note that permanent active longitudes have been found in other types of active stars (Jetsu et al. 1993; Berdyugina \& Tuominen 1998).

The basic concept of the single star model of RW Aur is that the longer period of $5-6$ days is the rotational period of the star, while the shorter one, about 2.77 , indicates that there are two active regions on the stellar surface two footpoints of the accretion columns on opposite sides of the star.

The following arguments support this concept. With $R_{*} \geq 1.3 R_{\odot}$ (Sect. 5 ) and $v \sin i=16 \mathrm{~km} \mathrm{~s}^{-1}$ the period must be $\geq 4$. 1 . That is, the observed 5-6 days period is more likely to be the true rotational period than the 2 d 77 period. A similar period of 5.4 was first found in variations of the $\mathrm{H} \beta$ profile in RW Aur A in observations of 1980-82 (Grinin et al. 1983). Variations in the broad emission lines formed in a large volume of the magnetosphere and the stellar wind reflect the rotational period, while the spectral features associated with the accretion processes closer to the star reflect the local structure of the accretion channels and may show period(s) shorter than the rotational one.

The phase-shift in the variations of the radial velocities and the EW of the He I narrow emission, about 0.25 , is a clear indication that the emission is formed in a spot-like area. The EW is larger when the spot is face-on to the observer, while the radial velocity is larger when the spot is near the limb. With two spots on opposite sides, this happens twice over one rotational period of about 5.5, hence the period of 2.77 appears in the data.

The unexpected result of our research is the absence of any correlation between veiling and brightness, although both parameters were changing over wide ranges. The considerable increase of veiling at constant brightness of the star (Fig. 12) excludes the possibility that the veiling was caused by a rise in the additional continuum radiated by a hot spot (unless the increased brightness was precisely balanced by increased circumstellar extinction).

Besides the hot continuum, the veiling can be raised by narrow emission lines of neutral metals which partially fill in the photospheric absorptions. Variability in the strength of these hypothetical narrow emissions could

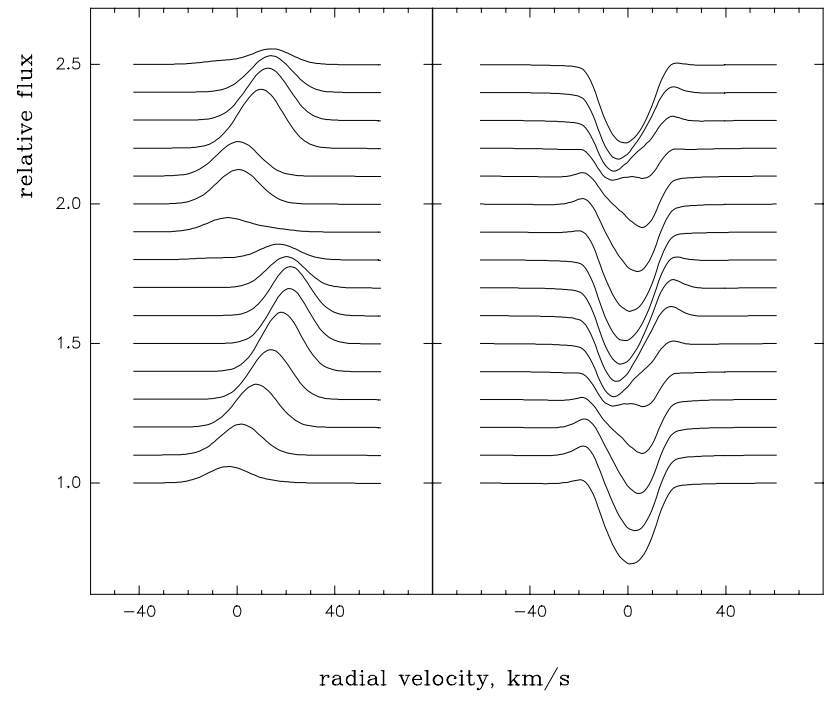

Fig. 26. Variations of emission and absorption line profiles in the spectrum of the spotted star (shown in Fig. 25), integrated over the stellar surface. Each spectrum corresponds to a certain phase of the axial rotation; step in phase $=1 / 16$

cause variability of the veiling even at constant brightness of the star. An argument in favour of such an interpretation can be found in the spectrum taken at the highest value of the veiling (HJD 2450382.5). As was written in Sect. 3, narrow absorption components are usually present on top of many broad emission lines of neutral metals. In the spectrum taken at highest veiling, these narrow absorptions turned into narrow emissions (Fig. 9). The radial velocities of these narrow emissions of the metals correspond to that of the He I narrow emission. We may suggest that the narrow emissions of the metals form in a chromosphere-like region associated with the footpoint of the accretion column. Since we know already that the radial velocity of the He I narrow emission varies periodically, we may expect that the narrow emissions of the metals also vary in radial velocity, and this must be reflected in the variability of the photospheric line profiles distorted by these narrow emissions.

In order to check this hypothesis numerically, we calculated a synthetic spectrum from a single rotating star with two spots of "chromospheric" emission. Figure 25 shows the positions of the two spots as they are projected onto the stellar disk at different phases during the rotation of the star. Figure 26 shows an example of line profile variations in an absorption line partially filled in with the chromospheric emission, and in a pure emission line (like He I). As the star rotates, the radial velocities of the absorption line vary in anti-phase with respect to those of the emission line, and the EWs of the emission vary with a phase-shift to the radial velocity, similar to that observed in RW Aur. The observed amplitudes and phase shifts can be reproduced with two spots of about 20 degrees in radius, located at latitudes of $\pm 45^{\circ}$ at opposite longitudes, as is shown in Fig. 25. With an inclined magnetic axis the spots may be close to the magnetic poles. One may notice, 
however, that the velocity variations in such a model are not quite sinusoidal. Spectra with higher signal-to-noise ratios are needed to check whether the predicted absorption line distortions shown in Fig. 26 are really present. Although the hypothesis of the chromospheric emissions filling in the photospheric lines accounts for most of the observed variations, it is not clear why these emissions were never seen above the continuum level in all the photospheric lines.

The hot spots in CTTSs are not as long-living as the cool spots related to the local magnetic fields, i.e. accretion processes are not stable. Contrary to the case of a binary star, the variations in the radial velocities caused by the spot effect described above may disappear sometimes. This could be an observational test to distinguish between the two models.

\section{Conclusions}

Our spectroscopic and photometric observations of the unusual T Tauri star RW Aur A have revealed extremely complex patterns of variability in all emission and absorption lines, both with regard to line profiles, equivalent widths/fluxes and radial velocities of the spectral components. A number of these components vary with a period of 2.77 . The variations are regular, and indicate that these patterns have been stable over several years.

In Paper I we reported the discovery of periodic, sinusoidal, small amplitude radial velocity changes in the weak spectrum of narrow absorption lines (WALs). Our new observations in Nov. 1999 confirm the period, phase and amplitude of the WAL velocities. Relative to these, the narrow emission lines of He vary in anti-phase. The broad emission lines, dominating in the spectrum of the star, show also the double period of 5-6 days. The veiling shows no corelation with brightness, although both parameters vary in a wide range. This is partly due to additional variable opacity of the layers above the photosphere (the shell), which imitates lower veiling and also makes the spectrum of RW Aur A vary in spectroscopic luminosity. The presence of chromospheric-like line emission in the photospheric lines most likely also plays a role. In one night we observed a spectrum of RW Aur A with exceptionally strong shell and accretion components present in many lines of neutrals and ions, which makes it possible to estimate the physical conditions in different parts of the accretion stream.

In order to account for all the periodic phenomena, we present two sketches of models, both dependent on the presence of non-axisymmetric accretion.

The first model is based on the binary hypothesis, where the secondary is assumed to generate enhanced accretion from one region of the disk, from where the gas stream originates and finally hits the surface of the primary. With new data on the inclination of the jet (Dougados, private communication), which is presumably aligned with the orbital axis, we get a solution where the secondary is a brown dwarf moving at a distance of only
8-10 $R_{\odot}$ from the primary in a nearly circular orbit. The presence of this secondary is considered to be responsible for the exceptional properties of RW Aur compared to other CTTSs.

The second model assumes that RW Aur A is a single star with two major accretion streams within a global magnetosphere that is tilted relative to the rotation axis or is intinsically non-axisymmetric.

Both these model concepts can explain quantitatively and qualitativly most of the complex variations, but both also have their short-comings. These ideas are intended to provide a basis for further developments of the theoretical concepts, and also for selecting key observational tests.

Acknowledgements. Thanks go to E. A. Kolotilov who kindly provided the $U B V$ magnitudes of comparison stars used in our CCD photometry of RW Aur, to David Kennedal who made the $U B V$ photometry at the Swedish $60 \mathrm{~cm}$ telescope, and to P. Artymowicz and P. Carlqvist for enlightening discussions. We also thank Catherine Dougados for providing information on the jet prior to publication. This work was supported by the Academy of Finland, the Finnish Graduate School of Astronomy and Space Physics, the Crafoord Foundation, the Swedish Natural Science Foundation and the Portuguese foundation for Science and Technology.

\section{References}

Appenzeller, I., \& Wolf, B. 1982, A\&A, 105, 313

Appenzeller, I., Östreicher, R., Schiffer, J. G., Egge, K. E., \& Pettersen, B. R. 1983, A\&A, 118, 75

Artymowicz, P., \& Lubow, S. H. 1996, in Disks and outflows around young stars, ed. S. Beckwith, J. Staude, A. Quetz, \& A. Natta, Lecture Notes in Physics, 465, 115

Basri, G., Johns-Krull, C. M., \& Mathieu, R. D. 1997, AJ, 114, 781

Batalha, C. C., Stout-Batalha, N. M., Basri, G., \& Terra, M. A. O. 1996, ApJS, 103, 211

Berdyugina, S. V., \& Tuominen, I. 1998, A\&A, 336, L25

Calvet, N. 1998, in Accretion processes in Astrophysical Systems: Some Like it Hot!, ed. Holt, \& Kallman, American Institute of Physics

Chelli, A., Carrasco, L., Mújica, R., Recillas, E., \& Bouvier, J. 1999, A\&A, 345, L9

Cohen, M., \& Schwartz, R. D. 1976, MNRAS, 174, 137

Dougados, C., Cabrit, S., Lavalley, C., \& Ménard, F. 2000, A\&A, in press

Elias, J. H. 1978, ApJ, 224, 857

Errico, L., Lamzin, S. A., \& Vittone, A. A. 2000, A\&A, 357, 951

Gahm, G. F. 1970, ApJ, 160, 1117

Gahm, G. F., Gullbring, E., Fischerström, C., Lindroos, K. P., \& Lodén, K. 1993, A\&AS, 100, 371

Gahm, G. F., Lodén, K., Gullbring, E., \& Hartstein, D. 1995, A\&A, 301, 89

Gahm, G. F., Petrov, P. P., Duemmler, R., Gameiro, J. F., \& Lago, M. T. V. T. 1999, A\&A, 352, L95 (Paper I)

Ghez, A. M., Neugebauer, G., \& Matthews, K. 1993, AJ, 106, 2005

Ghez, A. M., White, R. J., \& Simon, R. 1997, ApJ, 490, 353

Giampapa, M. S., Basri, G. S., Johns, C. M., \& Imhoff, C. L. 1993, ApJS, 89, 321 
Grinin, V. P., Petrov, P. P., \& Shakhovskaya, N. I. 1983, in Activity in Red Dwarf Stars, ed. P. B. Byrne, \& M. Rodonò (Reidel, Dordrecht), 513

Gullbring, E., Barwig, H., Chen, P. S., Gahm, G. F., \& Bao, M. X. 1996, A\&A, 307, 791

Hamann, F. 1984, ApJS, 93, 485

Hartigan, P., Hartmann, L., Kenyon, S., Hewett, R., \& Stauffer, J. 1989, ApJS, 70, 899

Hartmann, L. 1982, ApJS, 48, 109

Hartmann, L. 1998, Accretion processes in star formation (University of Cambridge Press)

Hartmann, L., Hewett, R., Stahler, S., \& Mathieu, R. D. 1986, ApJ, 309, 275

Herbig, G. H., \& Soderblom, D. R. 1980, ApJ, 242, 628

Herbst, W., Herbst, D. K., \& Grossman, E. J. 1994, AJ, 108, 1906

Hessman, F. V., \& Guenther, E. W. 1997, A\&A, 321, 497

Ilyin, I. V. 2000, High resolution SOFIN CCD échelle spectroscopy, Ph.D. Thesis, University of Oulu
Jetsu, L., Pelt, J., \& Tuominen, I. 1993, A\&A, 278, 449

Johns-Krull, C. M., Valenti, J. A., Hatzes, A. P., \& Kanaan, A. 1999, ApJ, 510, L41

Joy, A. H. 1945, ApJ, 102, 207

Königl, A. 1991, ApJ, 370, L39

Mundt, R., \& Eislöffel, J. 1998, ApJ, 116, 860

Mundt, R., \& Giampapa, M. S. 1982, ApJ, 256, 156

Muzerolle, J., Hartmann, L., \& Calvet, N. 1998, AJ, 116, 455

Palla, F., \& Stahler, S. W. 1999, ApJ, 525, 772

Petrov, P. P., Gullbring, E., Ilyin, I., et al. 1996, A\&A, 314, 821

Rutledge, R. E., Basri, G., Martin, E. L., \& Bildsten, L. 2000, ApJ, 538, L141

Stout-Batalha, N. M., Batalha, C. C., \& Basri, G. S. 2000, ApJ, 532, 474

Tuominen, I., Ilyin, I., \& Petrov, P. 1999, in Astrophysics with the NOT, ed. H. Karttunen, \& V. Piirola, Univ. Turku, 47

Valenti, J. A., Basri, G., \& Johns, C. M. 1993, AJ, 106, 2024 